\title{
WHEEL/RAIL CONTACT STRESS EVALUATION BY MEANS OF THE MODIFIED STRIP METHOD
}

The Strip Method is often used for rail/wheel contact area and contact normal stress evaluation. The paper deals with the computational time saving procedure when the computation accuracy is guaranteed. We included the local coordinate system with a presupposed semi-circular course of the normal stress for the purpose of the integral that is necessary for deformation in the middle of individual strips evaluation. The integral is solvable analytically. The input parameters for individual parts of splines and individual strips expression are possible to insert after analytical solution.

Keywords: Wheel/rail contact, contact stress evaluation, modified Strip method, optimized computation procedure.

\section{Introduction}

An important parameter influencing the power effect of a wheel on the rail is the size and shape of the contact area as well as the normal stress distribution which has the impact on it. Nowadays various methods are used to find out the size of contact areas and stresses [1,2]. It is necessary to mention the Hertz method as one of the oldest up to date used methods. It provides acceptable results for a large area in spite of many simplifications. Another computational procedure is the Kalker variation method or the strip method which is, thanks to its results, close to reality and is used in the following calculations [3]. Nowadays, another group of calculation program systems (ANSYS, ADINA) is used in certain situations. The systems work on the base of finite elements method theory.

\section{Strip method}

The Strip Method presupposes quasi-static rolling [1]. The principal idea of the theory is to take into consideration slim contact areas in the $y$-direction. In Fig. 2 there are two bodies in contact. In fact the geometrical parameters (of railway wheel and rail) should be similar to the reality. The deformation zones are of the similar shapes too. In spite of this fact the parameters (the displacements $w_{1}$ and $w_{2}$ ) in Fig. 1 are rather different for better understanding of the theory.

In fact, the contact area should be a plane (parallel with the $\mathrm{x}-\mathrm{y}$ plane).

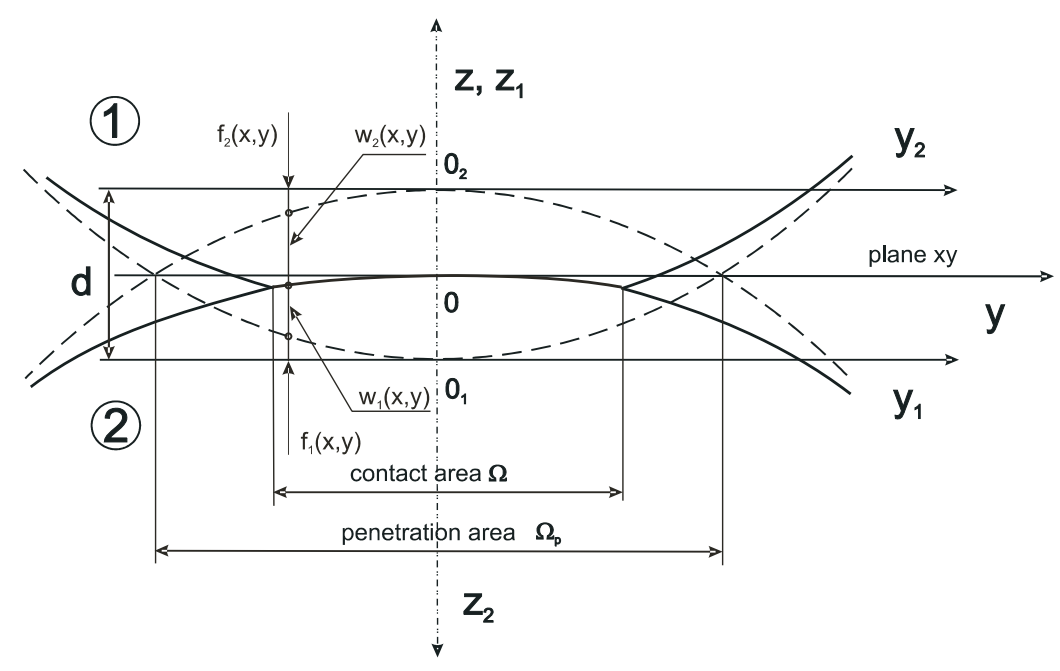

Fig. 1 Variation Coordinate body system at the contact

\footnotetext{
* Tomas Lack, Juraj Gerlici

Faculty of Mechanical Engineering, University of Zilina, Slovakia, E-mail: tomas.lack@fstroj.uniza.sk
} 
The method presupposes the existence of two rotating bodies 1 and 2 with surfaces $S 1$ and $S 2$. The bodies touch in the point 0 , which is at the same time the beginning of their spatial coordinate systems. The axes $x$ and $y$ determine the horizontal base. We will mark the horizontal coordinate as the $z$ - axis. If there is no influence of a normal force $Q$, then there exclusively exists geometrical binding between the bodies.

If the bodies are pressed against each other by the normal force $Q$, a deformation and a contact area $\Omega$ instead of a contact point arises between the bodies.

- The geometrical profile shape of the first body surface will be marked $f_{1}(x, y)$, the geometrical profile shape of the second body surface will be marked $f_{2}(x, y)$.

- The elastic displacement in the $z$-axis direction caused by the deformation of the first body surface will be marked $w_{1}(x, y)$, the displacement in the z-axis direction caused by the deformation of the second body surface will be marked $w_{2}(x, y)$.

- The displacement of bodies centers against each other in the axis-z direction will be marked $d$.

- The perpendicular distance between the points of the deformed bodies surfaces will be marked $\delta(x, y)$.

\section{Normal stress approximation over a strip}

The stress evaluation over the $\mathrm{k}^{\text {th }}$ strip (Fig. 2) is approximated in a standard way [4]:

$$
p_{k}\left(x, y_{k}\right)=p_{0 k} \cdot \sqrt{1-\left(\frac{x}{x_{d k}}\right)^{2}}
$$

where:

$p_{k}\left(x, y_{k}\right)$ normal stress in the $x$ position,

$p_{0 k} \quad$ maximum normal stress,

$x_{d k} \quad$ half length of the $\mathrm{k}^{\text {th }}$ strip.

We aimed our interest at increasing the computational effort. To obtain the requested results, deformations and stresses in the

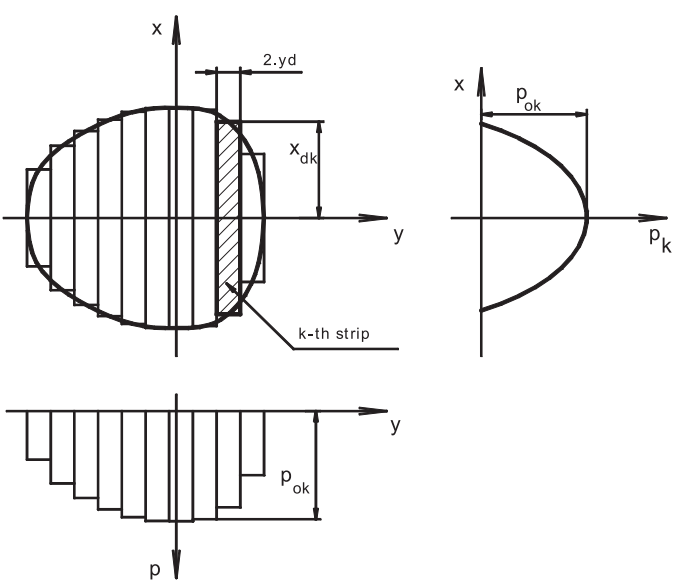

Fig. 2 Contact patch distribution into the strips, stress distribution over individual strips middle of strips, we utilized the procedures introduced in the next text.

\section{Time optimized computational procedure for contact stresses assessment}

The equation system assembly is necessary for the stress evaluation in the middle of strips $[4,5,6]$ :

$$
[M] \cdot\left\{p^{(0)}\right\}=\left\{w^{(0)}\right\}
$$

where:

$[M]$ influence coefficients matrix,

$\left\{p^{(0)}\right\}$ normal stresses in the middle of strips vector,

$\left\{w^{(0)}\right\}$ in the middle of strips strains vector.

The $[M]$ matrix elements are of values:

$$
M_{k, s}=H \cdot I_{k, s}^{(0)}
$$

where:

$$
H=\frac{1}{\pi} \cdot\left(\frac{1-\mu_{1}^{2}}{E_{1}}+\frac{1-\mu_{2}^{2}}{E_{2}}\right)
$$

where:

$\mu_{1}$ Poisson's ratio of a first body,

$\mu_{2}$ Poisson's ratio of a second body.

$E_{1}$ modulus of stiffness of a first body.

$E_{2}$ modulus of stiffness of a second body.

The vector elements are:

$$
w_{k}^{(0)}=z_{2 k}-z_{1 k}-D
$$

where:

$w_{k}^{(0)} \quad$ deformation in the middle of $\mathrm{k}^{\text {th }}$ strip.

$z_{1 k} \quad \mathrm{z}$ - coordinate of a first body in the middle of $\mathrm{k}^{\text {th }}$ strip,

$z_{2 k} \mathrm{z}$ - coordinate of second body in the middle of $\mathrm{k}^{\text {th }}$ strip,

$D$ straightening up of bodies.

The aim is to compute the value $I_{k s}^{(0)}$ in accordance with relation below:

$$
I_{k, s}^{(0)}=2 \cdot \int_{0}^{x_{d k}} \int_{y_{k k}}^{y_{r k}} \sqrt{\frac{1-\left(\frac{x}{x_{d k}}\right)^{2}}{x^{2}+\left(y_{s}-y\right)^{2}}} \cdot d y \cdot d x
$$

when:

$$
\begin{aligned}
& y_{l k}=y_{k}-y_{d} \\
& y_{r k}=y_{k}+y_{d}
\end{aligned}
$$

where:

$x_{d k}$ half length of the kth strip,

$y_{k}$ y-coordinate of kth strip, 
$y_{s} \quad \mathrm{y}$-coordinate of sth strip,

$y_{d}$ half width of strips.

This integral can be modified into one- dimension integral in the form:

$$
\begin{aligned}
& I_{k, s}^{(0)}=-\frac{2}{\left|x_{d k}\right|} \cdot \int_{0}^{x_{d k}} \sqrt{x_{d k}^{2}-x^{2}} \cdot \operatorname{Ln}\{A A\} \cdot d x \\
& A A=\frac{[A B] \cdot[A C]}{x^{2}} \\
& A B=\sqrt{x^{2}+\left(y_{s}-y_{r k}\right)^{2}}+y_{s}-y_{r k} \\
& A C=\sqrt{x^{2}+\left(y_{s}-y_{l k}\right)^{2}}-y_{s}+y_{l k}
\end{aligned}
$$

It is unavoidable to solve the integral in the relation (9) numerically, because it has no analytical solution. This computation is very time consuming for separate strips in the relationship to other strips.

This is the reason for the new procedure development that is not dependent on a real strip length. We presuppose that a strip is of one unit length. Description of individual parameters in the $\mathrm{x}, \mathrm{y}$ coordinate system is in Fig. 3 and a description of individual parameters in the $u, v$ coordinate system is in Fig. 4 .

For the value $I_{k, s}^{(0)}$ the relation is valid:

$$
I_{k, s}^{(0)}=i_{k, s}^{(0)} \cdot x_{d k}
$$

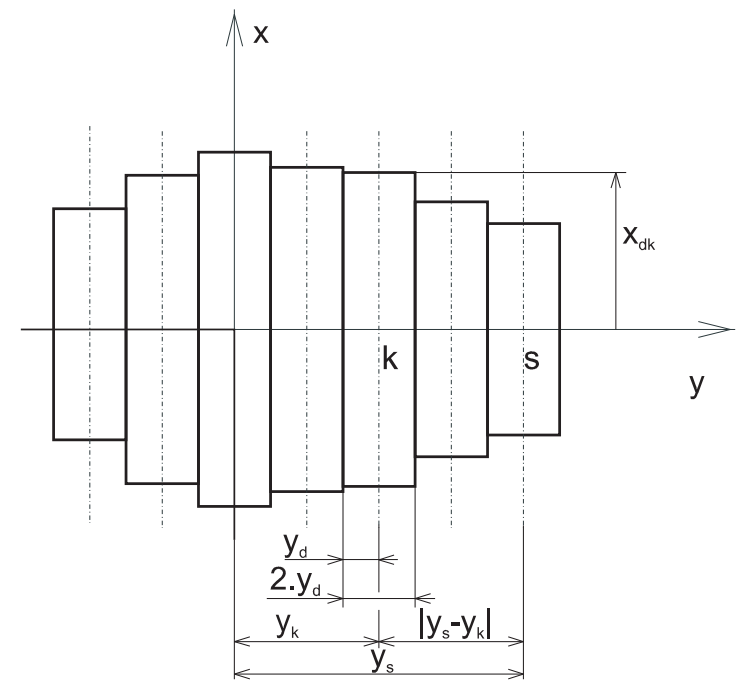

Fig. 3 Description of individual parameters in the coordinate system

We establish:

- the $\mathrm{k}^{\text {th }}$ strip length in the $u, v$ system has the value of 1

- the $\mathrm{k}^{\text {th }}$ strip width in the $u, v$ system has the value:

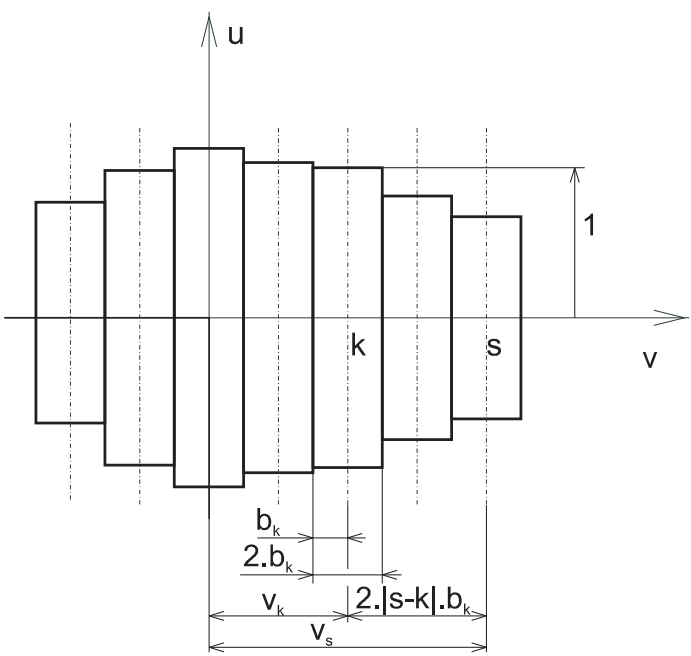

Fig. 4 Description of individual parameters in the coordinate system

$$
b_{k}=\frac{y_{d}}{x_{d k}}
$$

The relationship for the $I_{k s}^{(0)}$ computation is valid:

$$
I_{k, s}^{(0)}=2 \cdot \int_{0}^{1} \int_{-1}^{1} \sqrt{\frac{1-u^{2}}{u^{2}+\left[(2 \cdot|s-k|-v) \cdot b_{k}\right]^{2}}} \cdot d v \cdot d u(15)
$$

The value: " $i$ " of this integral or value: " $E$ " of decimal logarithm of integral are depicted in graphs in Figs. 5 - 10.

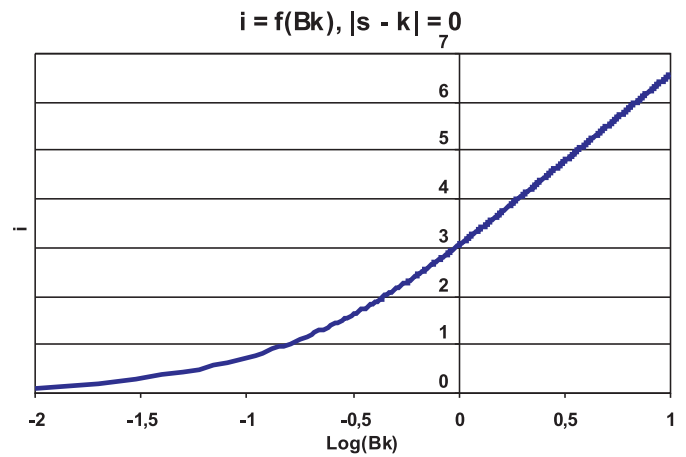

Fig. 5 The integral value " $i$ " in dependence on $\log (B k)$ on condition that $|s-k|=0$

The value of an integral decimal logarithm " $E$ " can be expressed via the polynomial of 6 -th grade:

$$
\left.E=\left(\left(\begin{array}{l}
\left.\left(\left(a_{6} \cdot L+a_{5}\right) \cdot L+a_{4}\right) \cdot\right) \\
\left(L+a_{3}\right. \\
L+a_{1}
\end{array}\right) \cdot L+a_{2}\right) \cdot\right) \cdot L+a_{0}
$$




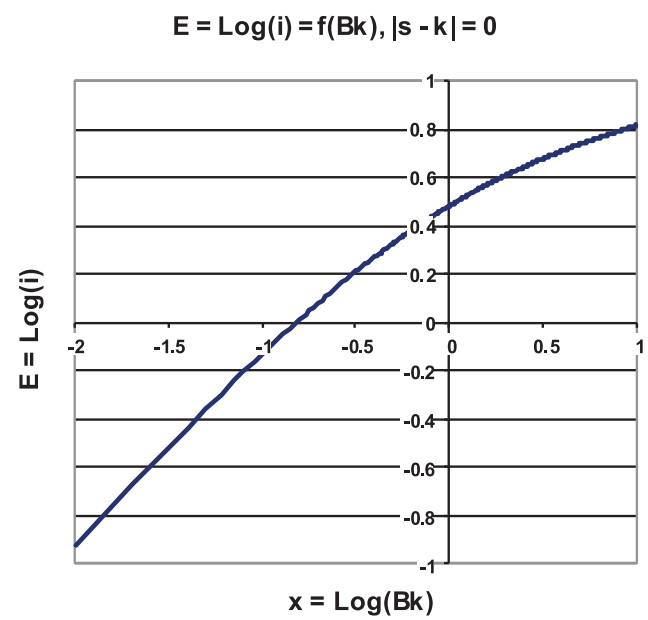

Fig. 6 The integral decimal logarithm value " $E$ " in dependence on $t$ $\log (B k)$, on condition that $|s-k|=0$

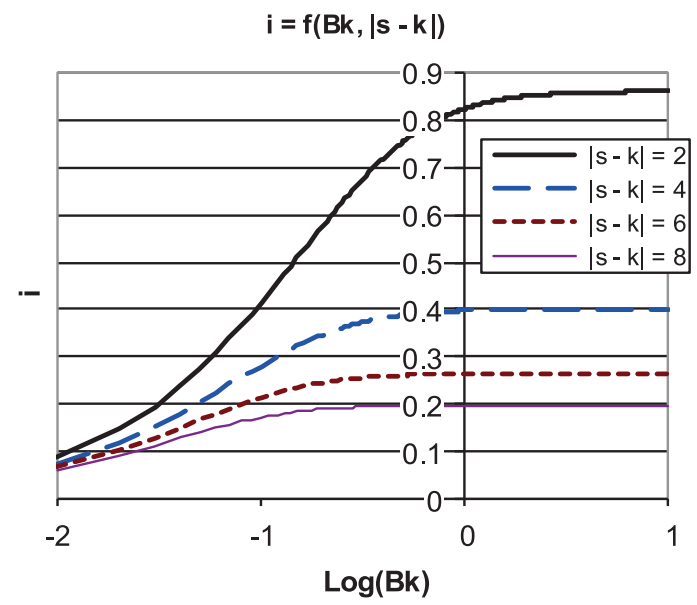

Fig. 7 The integral value " $i$ " in dependence on $\log (B k)$ on condition that $|s-k|=\langle 2 ; 8\rangle$

$E=\log (i)=f(B k,|s-k|)$

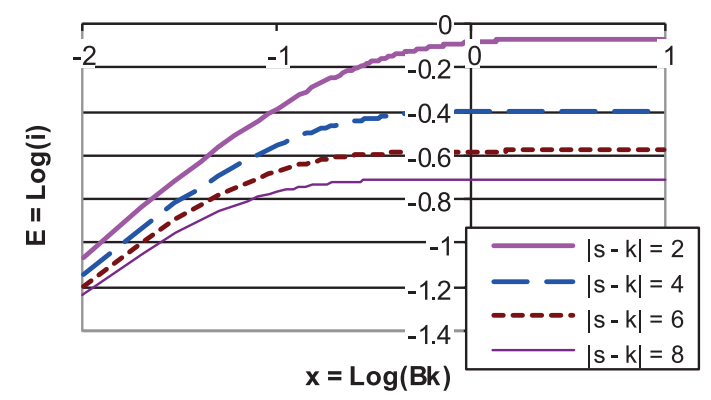

Fig. 8 The integral decimal logarithm value " $E$ " in dependence on $\log (B k)$ on condition that $|s-k|=\langle 2 ; 8\rangle$

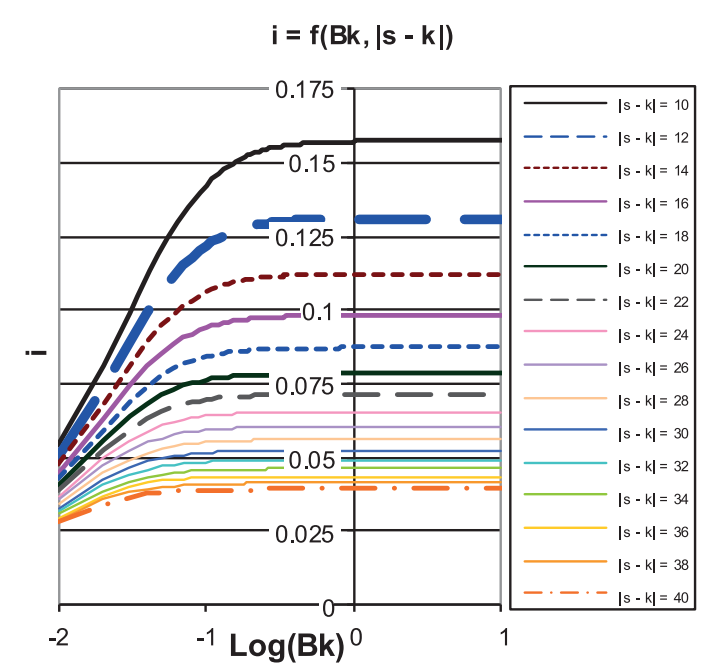

Fig. 9 The integral value " $i$ " in dependence on $\log (B k)$ on condition that $|s-k|=\langle 10 ; 40\rangle$

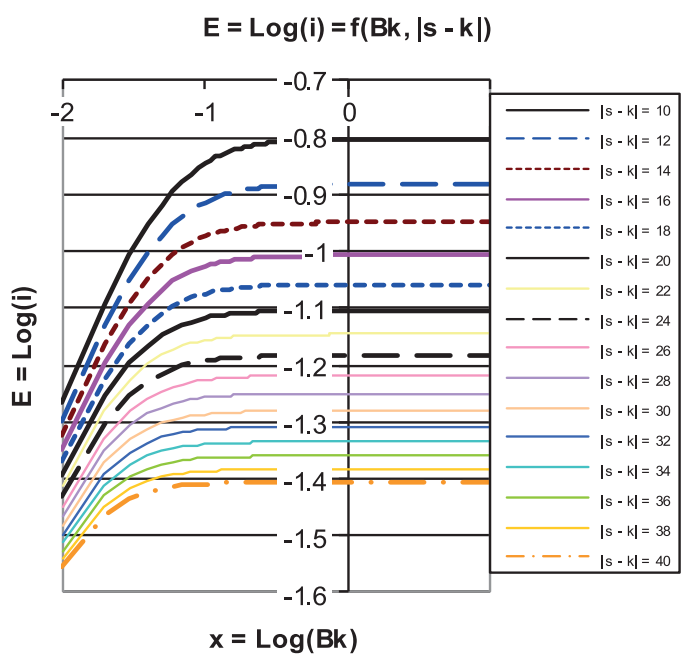

Fig. 10 The value of integral decimal logarithm in dependence on $\log (B k)$ on condition that $|s-k|=\langle 10 ; 40\rangle$

$$
L=\log \left(b_{k}\right)
$$

Polynomial coefficients from $a_{6}$ to $a_{3}$ are in Table 1 .

Polynomial coefficients from to are in Table 2.

The $i_{k, s}^{(0)}$ value can be expressed via this relation:

$i_{k, s}^{(0)} \approx 10^{E}$ 
Polynomial coefficients $a_{6}$ to $a_{3}$

\begin{tabular}{|c|c|c|c|c|}
\hline$|s-k|$ & $a_{6}$ & $a_{5}$ & $a_{4}$ & $a_{3}$ \\
\hline 0 & -0.00255 & -0.001497 & 0.022573 & 0.009238 \\
\hline 2 & -0.00756 & -0.026714 & 0.015249 & 0.105083 \\
\hline 4 & 0.003839 & -0.008085 & -0.02931 & 0.068803 \\
\hline 6 & 0.007608 & 0.004252 & -0.03718 & 0.037078 \\
\hline 8 & 0.007902 & 0.009316 & -0.03513 & 0.019712 \\
\hline 10 & 0.007030 & 0.01098 & -0.03057 & 0.010126 \\
\hline 12 & 0.005830 & 0.011066 & -0.02574 & 0.004737 \\
\hline 14 & 0.004628 & 0.010462 & -0.0213 & 0.001656 \\
\hline 16 & 0.003549 & 0.009613 & -0.01744 & -0.00014 \\
\hline 18 & 0.002611 & 0.008678 & -0.01415 & -0.00115 \\
\hline 20 & 0.001806 & 0.007735 & -0.01138 & -0.00165 \\
\hline 22 & 0.001127 & 0.006837 & -0.00907 & -0.00186 \\
\hline 24 & 0.000534 & 0.005942 & -0.00714 & -0.00182 \\
\hline 26 & 0.000064 & 0.005204 & -0.00551 & -0.00174 \\
\hline 28 & -0.000320 & 0.00455 & -0.00417 & -0.00164 \\
\hline 30 & -0.00063 & 0.003985 & -0.00305 & -0.00152 \\
\hline 32 & -0.00087 & 0.003496 & -0.0021 & -0.00139 \\
\hline 34 & -000102 & 0.003193 & -0.00132 & -0.00141 \\
\hline 36 & -0.00128 & 0.002579 & -0.00064 & -0.00097 \\
\hline 38 & -0.0014 & 0.002248 & $-7,3 \mathrm{E}-05$ & -0.00083 \\
\hline 40 & -0.00151 & 0.001912 & 0.000334 & -0.00069 \\
\hline & & & & \\
\hline
\end{tabular}

For a strip with the coordinate $x$ deformation computation the following relation is valid:

$$
w_{k}(x)=H \cdot \sum_{s=1}^{n} p_{s}^{(0)} \cdot I_{k, s}(x),
$$

where integral:

$$
I_{k, s}\left(x_{k}\right)=\int_{-x_{d k}}^{x_{d, k}} \int_{y_{l k}}^{y_{p, k}} \sqrt{\frac{1\left(\frac{x}{x_{d k}}\right)^{2}}{\left(x_{k}-x\right)^{2}+\left(y_{s}-y\right)^{2}}} \cdot d y \cdot d x
$$

This integral can be modified into the one-dimensional integral in form:

$$
\begin{aligned}
& I_{k, s}\left(x_{k}\right)=-\frac{1}{\left|x_{d k}\right|} \cdot \int_{-x_{d k}}^{x_{d k}} \sqrt{x_{d k}^{2}-x^{2}} . \\
& \cdot \operatorname{Ln}\left\{\frac{\sqrt{\left(x-x_{k}\right)^{2}+\left(y_{s}-y_{l k}\right)^{2}}-y_{s}-y_{l k}}{\sqrt{\left(x-x_{k}\right)^{2}+\left(y_{s}-y_{r k}\right)^{2}}-y_{s}-y_{r k}}\right\} \cdot d x
\end{aligned}
$$

This integral has no analytical solution, so it is unavoidable to compute this integral numerically. In relation to other strips, the mentioned solution for strip by strip computation is time con-

\begin{tabular}{|c|c|c|c|}
\hline$|s-k|$ & $a_{2}$ & $a_{1}$ & $a_{0}$ \\
\hline 0 & -0.16037 & 0.467393 & 0.483852 \\
\hline 2 & -0.14821 & 0.079591 & -0.08251 \\
\hline 4 & -0.04814 & 0.015369 & -0.39959 \\
\hline 6 & -0.0153 & 0.005056 & -0.5794 \\
\hline 8 & -0.0027 & 0.00233 & -0.7058 \\
\hline 10 & 0.00236 & 0.001448 & -0.80338 \\
\hline 12 & 0.004248 & 0.001116 & -0.88289 \\
\hline 14 & 0.004707 & 0.000973 & -0.95 \\
\hline 16 & 0.004506 & 0.000906 & -1.00807 \\
\hline 18 & 0.004019 & 0.000859 & -1.05925 \\
\hline 20 & 0.003431 & 0.000813 & -1.10502 \\
\hline 22 & 0.002834 & 0.000765 & -1.1464 \\
\hline 24 & 0.002282 & 0.000698 & -1.18418 \\
\hline 26 & 0.001758 & 0.000651 & -1.21892 \\
\hline 28 & 0.001302 & 0.000611 & -1.25109 \\
\hline 30 & 0.0009 & 0.000577 & -1.28104 \\
\hline 32 & 0.000545 & 0.000544 & -1.30905 \\
\hline 34 & 0.000223 & 0.000551 & -1.33537 \\
\hline 36 & -0.00002 & 0.000445 & -1.36018 \\
\hline 38 & -0.00026 & 0.000409 & -1.38364 \\
\hline 40 & -0.0004 & 0.000389 & -1.40591 \\
\hline
\end{tabular}
suming.
Polynomial coefficients $a_{2}$ to $a_{0}$

Table 2

We establish:

- the k-th strip length in the $u, v$ system has the value of 1

- the k-th strip width in the $u, v$ system has the value:

$$
b_{k}=\frac{y_{d}}{x_{d k}}
$$

and

$$
u_{k}=\frac{x_{k}}{x_{d k}}
$$

where:

$x_{k} x$ - coordinate, in the $x, y$ coordinate system on $\mathrm{k}^{\text {th }}$ strip,

$x_{d k}$ length of the $\mathrm{k}^{\text {th }}$ strip,

$u_{k} \quad u$ - coordinate, in the $u, v$ coordinate system on $\mathrm{k}^{\text {th }}$ strip.

$$
\begin{aligned}
& I_{k, s}\left(x_{k}\right)=i_{k, s}\left(u_{k}\right) \cdot x_{d k}, \\
& i_{k, s}\left(u_{k}\right)=i_{k, s}^{0} \cdot \frac{q_{k, s}\left(u_{k}\right)+q_{k, s}\left(-u_{k}\right)}{q_{k, s}^{(0)}}, \\
& q_{k, s}^{(0)}=2 \cdot \int_{0}^{1} \int_{-1}^{1} \frac{1-u^{2}}{\sqrt{u^{2}+\left[(2 \cdot|s-k|-v) \cdot b_{k}\right]^{2}}} \cdot d v \cdot d u(26)
\end{aligned}
$$




$$
q_{k, s}^{(0)}(\chi)=\int_{0}^{1} \int_{-1}^{1} \frac{1-u^{2}}{\sqrt{(\chi-u)^{2}+\left[(2 \cdot|s-k|-v) \cdot b_{k}\right]^{2}}} \cdot d v \cdot d u
$$

The $q_{k, s}^{(0)}$ integral values and $q_{k, s}(\chi)$ integral are utterably analytical.

Further, we compare the computational precision and computation speed gained by the Kalker's method with the results gathered by other methods.

\section{Computation results obtained by different methods}

In the following graphs are maximum stresses in contact courses against lateral shift of the wheel along a rail head movement from a wheel rim (Fig. 11), maximum stresses in contact courses against lateral shift of the wheel along a rail head movement to a wheel rim (Fig. 12) as well as the contact patch values courses against lateral shift of the wheel along a rail head movement from a wheel rim (Fig. 13) and contact patch area courses against lateral shift of the wheel along a rail head movement to a wheel rim (Fig. 14).
The computational time spent on the P5 computer, with 2GB RAMM, $3 \mathrm{GHz}$ frequency.

Utilized methods:

- Strip method level of tenth of second

- Kalker method with input of strip method results - level of second

- Kalker variation method - level of more than ten seconds.

\section{Conclusions}

The Strip Method is often used for rail/wheel contact area and contact normal stress evaluation. It presupposes quasi-static rolling. The principal idea of the theory is to take into consideration slim contact areas in the y-direction. The paper deals with the computational time saving procedure when the computation accuracy is guaranteed.

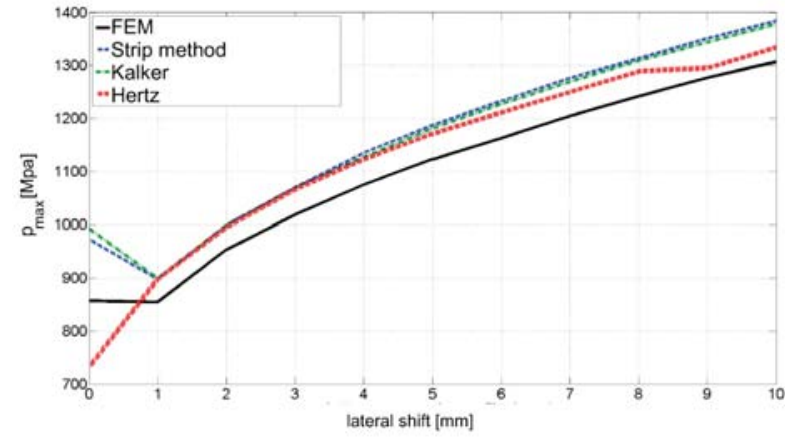

Fig. 11 Maximum stresses (pmax) in contact courses against lateral shift of the wheel tread along a rail head movement from a wheel rim

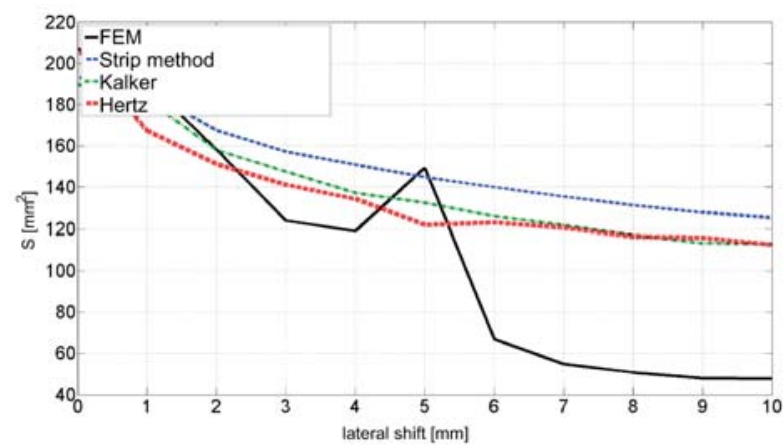

Fig. 13 Contact patch values (S) courses against lateral shift of the wheel tread along a rail head, movement from a wheel rim

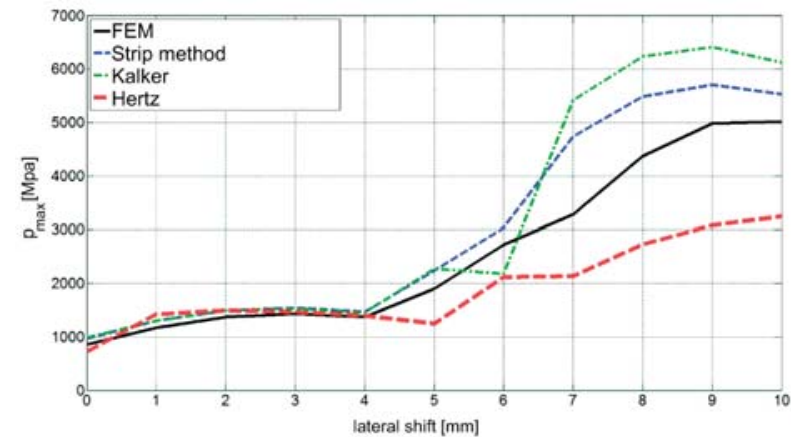

Fig. 12 Maximum stresses (pmax) in contact courses against lateral shift of the wheel tread along a rail head movement to a wheel rim

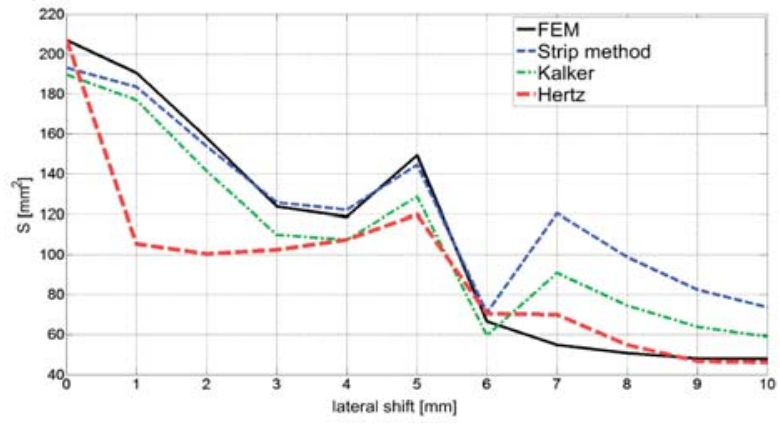

Fig. 14 Contact patch values (S) courses against lateral shift of the wheel tread along a rail head, movement to a wheel rim 
The introduced method enables contact patches and contact stresses between railway wheel and rail [7] under decreased computational time consumption. Rules and procedures characteristic of the Strip method are preserved. The stress computation acceleration is in this case based on the algorithm for numerical solution of integrals. We included the local coordinate system with a presupposed semi-circular course of the normal stress for the purpose of the integral computation. This integral computation is needed for deformation in the middle of individual strips evaluation. The integral is solvable analytically. The input values for the separate spline parts and separate strips computation are possible to insert after analytical solution of the integral. The procedure application may bring the practical benefit for researchers and computation - analysis experts who are interested in the field of vehicle dynamics simulations, rail / wheel contact analysis, as well as new profiles on the base of geometric characteristics design [8].

\section{Acknowledgements}

This paper was created during the processing of the project "RAILBCOT - RAIL Vehicles Brake COmponents Test Stand", ITMS Code 26220220011 based on the support of Research and Development Operational Program financed by European Fund of a Regional Development. The work was also supported by the Scientific Grant Agency of the Ministry of Education of the Slovak Republic and the Slovak Academy of Sciences in project No. 1/0347/12: "Railway wheel tread profile wear research under the rail vehicle in operation conditions simulation on the test bench.", project No. 1/0383/12: "The rail vehicle running properties research with the help of a computer simulation." and the project No. APVV-0842-11: "Equivalent railway operation load simulator on the roller rig.

\section{References}

[1] GERLICI J., LACK, T. et al.: Transport Means Properties Analysis - part I, p. 214, EDIS: University of Zilina, 2005, ISBN 80-8070408-2, 2005

[2] LACK, T. GERLICI, J.: Contact Area and Normal Stress Determination on Railway Wheel / Rail Contact, Communications - Scientific Letters of the University of Zilina, vol. 7, No. 2, 2005, pp. 38-45.

[3] SHACKLETON, P., IWNICKY, S.: Comparison of Wheel-rail Contact Codes for Railway Vehicle Simulation: An Introduction to the Manchester Contact Benchmark and Initial Results. Vehicle System Dynamics, vol. 46, No. 1-2, 2008, pp. 129-149.

[4] GERLICI, J., LACK, T.: Strip Method Modification for Stresses in a Wheel / Rail Contact Computation (in Slovak), Proc. of the 7th Intern. Conference Dynamics of Rigid and Deformable Bodies, Usti nad Labem, September, 2009.

[5] LACK, T., GERLICI, J.: Contact Stresses between Railway Wheel and Rail Computation by Means of Strip Method Optimized Procedure (in Slovak), Proc. of the $9^{\text {th }}$ Intern. Conference Dynamics of Rigid and Deformable Bodies 2011, Usti nad Labem, October, 2011.

[6] LACK, T., GERLICI, J.: Modified Strip Method Utilization for Wheel /Rail Contact Stress Evaluation, Proc. of the $9^{\text {th }}$ Intern. Conference on Contact Mechanics and Wear of Rail/Wheel Systems (CM2012), Chengdu, China, 2012.

[7] GERLICI, J., LACK, T.: Contact Geometry Influence on the Rail / Wheel Surface Stress Distribution, Proc. Engineering, vol. 2, No. 1, pp. 2249-2257, 2010.

[8] GERLICI, J., LACK, T.: Railway Wheel and Rail Head Profiles Development Based on the Geometric Characteristics Shapes. Wear, vol. 271, No. 1-2, Special issue, pp. 246-258, 2011. 